\title{
Booḱ reviews
}

who present late and have persistent pain. Trauma and tumours are included in the text.

Overall the book is well presented with good illustrations and useful references. It will be read by orthopacdic surgeons in training and indeed by consultants who do not have large children's practices. All the contributors are orthopaedic surgeons and a more rounded picture could have been achieved if there had been one chapter by a rheumatologist with paediatric expertise as there are a number of occasions where a medical opinion is invaluable. Overall the editor is to be congratulated.

I. KI.ENERMAN

Child Health Clinics. By Margaret Barker and Jonathan Ferrier. Pp 120: $£ 9.50$ paperback. John Wiley and Sons Ltd, 1988. ISBN (0-471-91626-9.

This book is designed as a simple introduction to the work of the child health clinic. It is targeted at general practitioners, clinical medical officers, and junior hospital paediatricians. It covers the general features of child health surveillance and the management of the types of common physical, social, emotional, and behavioural problems that present in the child health clinic.

While there is every virtue in being simple. I feel that the book will leave the reader to underestimate the level of clinical skill and knowledge that is required. For example, under the section of hearing testing, there is no emphasis on the level of clinical training required to do this competently. Under hearing testing and the role of the nurse in the clinic, it says that the clinic nurse can help with testing hearing, though again with no mention of the training that would be needed.

There are various other pieces of advice with which many of us would disagree. For example, the suggestion that all children whose growth curve is persistently below the 3 rd centile should be referred. Another example which many would disagree with was the statement that children with undescended testes should be referred by the age of 6 if possible.

Overall, this is a disappointing book. It lacks detail and there are many statements in it with which I would disagree, and many omissions of further information that $I$ would regard as important. The final chapters of the book covering child abuse and handicap I found much more helpful and provided a useful summary for the child health clinic doctor.

l. POLNAY

Overall, this book achieves its purpose. It provides several stimulating reviews in key topics, it is well written, very well referenced, and clearly indexed. It will be useful to interested haematologists and paediatricians dealing with neonatal problems. The excellent physiologic reviews, however, merit a wider readership.

P J DARBYSHIRE

Developmental and Neonatal Hematology. (Pediatric Hematology/Oncology Series). Edited by James A Stockman III and Carl Pochedly. Pp 348: $\$ 86 \cdot 50$ hardback. Raven Press, 1988. ISBN 0-88167-339-7.

This volume, written by a group of American authors, sets out to review current knowledge and document progress in the haematology of the fetus and neonate. It does not aim to provide comprehensive coverage of all aspects of the field, and the book suffers like many other multiauthor reviews from some degree of duplication. However, there are some excellent contributions within it.

Several areas of current interest are well dealt with by one or more authors. The chapters dealing with neonatal haemostasis and platelet function strike a good balance between the basic physiology and its clinical application. Erythropoiesis by Mark S Brown is similarly well structured, with chapters on fetal and neonatal erythropoiesis, followed by chapters dealing with physiologic anaemia of infancy and factors influencing its severity.

The review of blood component treatment points out clearly the risks of transfusion in the neonatal setting, and should be useful reading to anyone regularly involved in pacdiatric transfusion. Another highlight is the very thorough chapter on neonatal isoimmune thrombocytopenia by $\mathrm{V}$ Blanchette. It might, however, have benefitted from more discussion of the commoner problem of maternal and neonatal autoimmune thrombocytopenia somewhere in the volume.

On the negative side, the chapters on haemolytic disease of the newborn do have some duplication and one questions whether technical details of exchange transfusion techniques are appropriate in this setting.
Adult and Pediatric Urology. Edited by J Y Gillenwater, J T Grayhack, S S Howards, and J W Duckett. Pp 2095: £168 hardback (two volumes). Year Book Medical Publishers, 1987.

This book gives a broad, up to date and very thorough review of urology from 91 authors. Despite its large size (two parts, 2095 pages and $7.3 \mathrm{~kg}$ ) it is very readable. Each subject is comprehensively discussed, beginning with basic concepts and covering the relevant physiology, anatomy, and pathology as well as the detailed diagnosis and treatment.

The bulk of the book is defined as covering adult urology, with just over one quarter being devoted to paediatric practice. Much of the adult section, however, deals with general subjects that are highly relevant to paediatric urology. These include chapters that give general background information such as the principles of the various diagnostic imaging techniques, and chapters covering conditions such as calculus formation and management, trauma, renovascular hypertension. and bladder dysfunction.

Much of the paediatric section deals with congenital abnormalities and presents the different view points on the management of difficult problems, including the arguments for and against antenatal intervention for urological anomalies. It also deals with current concepts of managing vesicoureteric reflux and covers aspects of transplantation in children.

This book has drawn together a large amount of information and will be of value to paediatric surgeons and paediatric nephrologists and of interest to general paediatricians for occasional reference.

M G COULTHARD 